\title{
Implementation of an automated, real-time public health surveillance system linking emergency departments and health units: rationale and methodology
}

\author{
Kieran M. Moore, MD; ${ }^{*}$ Bronwen L. Edgar, MHSc; ${ }^{\dagger}$ Donald McGuinness, $\mathrm{MA}^{\dagger}$
}

\begin{abstract}
In September 2004, Kingston, Frontenac, Lennox and Addington (KFL\&A) Public Health, in collaboration with the Public Health Division of the Ontario Ministry of Health and Long-Term Care, Queen's University, the Public Health Agency of Canada, Kingston General Hospital and Hotel Dieu Hospital, began a 2-year pilot project to implement and evaluate an emergency department (ED) chief complaint syndromic surveillance system. Our objective was to evaluate a comprehensive and readily deployable real-time regional syndromic surveillance program and to determine its ability to detect gastrointestinal or respiratory outbreaks well in advance of traditional reporting systems. In order to implement the system, modifications were made to the University of Pittsburgh's Real-time Outbreak and Disease Surveillance (RODS) system, which has been successfully integrated into public health systems, and has enhanced communication and collaboration between them and EDs. This paper provides an overview of a RODS-based syndromic surveillance system as adapted for use at a public health unit in Kingston, Ontario. We summarize the technical specifications, privacy and security considerations, data capture, classification and management of the data streams, alerting and public health response. We hope that the modifications described here, including the addition of unique data streams, will provide a benchmark for future Canadian syndromic surveillance systems.
\end{abstract}

Keywords: syndromic surveillance, outbreak detection, public health informatics, emergency departments

\begin{abstract}
RÉSUMÉ
En septembre 2004, le Service de santé publique de Kingston, Frontenac, Lennox et Addington (KFL et A) a lancé, en collaboration avec la Division de la santé publique du ministère de la Santé et des Soins de longue durée de I'Ontario, I'Université Queen's, I'Agence de la santé publique du Canada, I'Hôpital général de Kingston et l'Hôpital Hôtel-Dieu, un projet pilote de deux ans afin d'implanter et d'évaluer un système de surveillance des syndromes liés aux principales plaintes dans les services d'urgence. Le projet visait à évaluer un programme régional en temps réel, facile à déployer et intégré de surveillance des syndromes et à déterminer sa capacité de protéger contre les éclosions de maladies gastro-intestinales ou respiratoires longtemps avant les systèmes
\end{abstract}

From the *Department of Emergency Medicine, Queen's University, Kingston, Ont., and the tEmergency Department Syndromic Surveillance Team, Kingston, Frontenac, and Lennox and Addington (KFL\&A) Public Health, Kingston, Ont.

Received: Apr. 17, 2007; revisions received: Sept. 27, 2007; accepted: Oct. 11, 2007

This article has been peer reviewed.

CJEM 2008;10(2):114-9 
habituels de déclaration. Afin de mettre en œuvre le système, on a modifié le système de surveillance des éclosions et des maladies en temps réel (RODS) de l'Université de Pittsburgh qui a intégré avec succès dans certains systèmes de santé publique où il a amélioré la communication et la collaboration entre ces systèmes et les services d'urgence. Cette communication présente un aperçu d'un système de surveillance des syndromes basé sur le système RODS et adapté à une unité de santé publique à Kingston (Ontario). Nous résumons les caractéristiques techniques, les considérations relatives à la vie privée et à la sécurité, la saisie des données, la classification et la gestion du cheminement des données, le déclenchement d'alertes et la réponse des services de santé publique. Nous espérons que les modifications décrites ici, y compris l'ajout de cheminements de données uniques, produiront un point de comparaison pour de futurs systèmes canadiens de surveillance des syndromes.

\section{Introduction}

\section{Rationale for syndromic surveillance}

The Walker Report, ${ }^{1}$ Naylor Report ${ }^{2}$ and the Campbell Commission Reports, ${ }^{3}$ among others, have pointed out severe gaps in the ability of the Canadian and Ontario public health systems to respond to infectious disease outbreaks. Improved surveillance capacity is a common theme. The Ontario Government's 3-year action plan to revitalize the public health system has focused on enhanced structure and support for health system preparedness. ${ }^{4}$ As one result, the Public Health Division of the Ministry of Health and Long-Term Care (MOHLTC) created a syndromic surveillance steering committee, which recommended a pilot site to investigate syndromic surveillance in Ontario.

Syndromic surveillance is an investigational approach in which health department staff employ automated data acquisition and statistical alarms to monitor disease indicators in real-time or near real-time, in order to detect disease outbreaks earlier than possible with traditional public health methods. ${ }^{5}$ The goal of this 2-year pilot, which began in September 2004, was to implement and evaluate an emergency department (ED) chief complaint syndromic surveillance system in collaboration with the Public Health Division of the MOHLTC; Kingston, Frontenac and Lennox \& Addington (KFL\&A) Public Health; Queen's University; the Public Health Agency of Canada; Kingston General Hospital; and Hotel Dieu Hospital. The University of Pittsburgh's Real-time Outbreak and Disease Surveillance (RODS, Version 3.0, University of Pittsburgh, Pittsburgh, Pennsylvania, 2004) was chosen as the best surveillance tool for the project because it was the only system with open-source software that permitted automated, electronic, real-time surveillance. ${ }^{6}$

RODS was developed for the US health care system with a specific focus on bioterrorism; therefore, substantial changes were necessary to tailor the tool to meet Canadian requirements. Major modifications were in the area of geospatial mapping, alert optimization, syndrome classification and the user interface. The differences between RODS 3.0 and the ED Syndromic Surveillance (EDSS) version of RODS, also known as RODS 3E, can be found in a technical evaluation report. ${ }^{7}$ RODS $3 \mathrm{E}$ receives, displays and analyzes ED visit data from 3 area hospitals within the KFL\&A Public Health jurisdiction and 4 hospitals in neighbouring Hastings \& Prince Edward Counties Health Unit. This represents approximately 250000 ED visits per year from a population of about 350000 people. The RODS architecture can potentially allow for the display and analysis of diverse data types from any number of public health jurisdictions.

\section{Privacy and confidentiality}

This pilot project adheres to all privacy policies and procedures of KFL\&A Public Health as well as to those of Ontario's new health care privacy legislation, the Personal Health Information Protection Act (PHIPA), which came into effect on Nov. 1, 2004. The act, designed to govern the collection, use and disclosure of personal health information within the health care sector, provides comprehensive and consistent rules to ensure that personal health information is kept confidential and secure.

In September 2004, in anticipation of PHIPA, the Kingston General and Hotel Dieu hospitals developed policies to ensure that all projects involving personal information would require a detailed business plan, privacy impact assessment and, where applicable, written approval from the Queen's University Research Ethics Board. Consequently, EDSS project directors, in consultation with legal council, developed a document entitled "Privacy and Confidentiality for Health Information for Emergency Department Chief Complaint, Syndromic Surveillance Privacy and Confidentiality Charter (September 2004)." This Charter contained an executive summary of the project as well as the principles, policies and procedures necessary to meet PHIPA requirements. A privacy impact assessment 
document was also prepared to address privacy issues relating to the project. Both documents were submitted to the appropriate hospital authorities for review and comments, and the EDSS project was subsequently approved by the Queens University Research Ethics Board.

In September 2005, one additional hospital within the KFL\&A jurisdiction, the Lennox and Addington County General Hospital, was added to the system. After obtaining necessary Medical Advisory Committee approval, 4 additional sites from the Quinte Health Care hospitals, located in neighbouring Hastings \& Prince Edward Counties Health Unit, were added to the system.

\section{Objectives of the Ontario syndromic surveillance pilot project}

EDSS has dual potential functions: to help public health units protect community health, and to facilitate the monitoring of ED volumes, ED admissions and surge capacity. The latter will enhance hospital responses to patient surges, particularly in the event of a disaster or pandemic influenza. The main objectives of this project were to determine whether real-time monitoring of ED patient chief complaints can assist in the early detection of gastrointestinal (GI) and respiratory outbreaks, to determine whether the surveillance tool can enhance public health investigation of disease outbreaks and to determine whether the tool can improve the capacity of public health professionals to respond to alerts of infectious disease outbreaks.

\section{Methods}

\section{Data collection and transmission}

The RODS public health surveillance data set includes 6 elements: chief complaint as entered by the triage nurse, date and time of visit, hospital name, patient age, patient sex and geographic identifier (e.g., postal code). These data are collected during ED registration or triage, requiring no additional workflow. They are then sent in HL7 format to the facility's HL7 message router, which forwards them to all appropriate systems. In RODS 3E, the 6 core data elements are complemented by the patient's Canadian Triage Acuity Score (CTAS), ${ }^{8,9}$ a 5-level ED triage scale based on chief complaint and first order modifiers such as vital sign abnormality, pain severity, comorbidity and mechanism of injury. ${ }^{10-12}$ In order to comply with PHIPA, the HL7 message router deletes personal identifiers and transmits the minimal data set to RODS.

The system captures additional real-time data from the 3 hospitals within the KFL\&A Public Health jurisdiction, including emergency and elective admissions, and positive febrile respiratory illness (FRI) screening results. ${ }^{13}$ Data elements captured for emergency and elective admissions are similar to those listed above for ED visits; however, admission diagnosis is captured in place of ED chief complaint. The FRI screening tool was introduced during the Severe Acute Respiratory Syndrome (SARS) outbreak in 2003, and all patients presenting to participating hospitals were screened for respiratory illness using a standard series of questions to identify new or worsening cough, shortness of breath, fever, shakes or chills in the last 24 hours. Patients who answered yes to these questions were considered FRI positive and appropriate infection control precautions were advised..$^{13}$

\section{Syndrome classification and alerts}

Current syndrome classification of the free text chief complaint is based on the Bayesian naive classification of chief complaints inherent in RODS (Complaint Coder $\mathrm{CoCo}$ ) as well as a subsequent classification using a maximum entropy classifier to minimize errors. ${ }^{14}$ The chief complaint field is used instead of the discharge diagnosis, which is often not coded on the chart for weeks and is not immediately available electronically in our settings. Prior studies have validated the use of respiratory and GI chief complaints, showing a high correlation with the discharge diagnosis..$^{15,16}$

The original RODS syndromes designed for bioterrorism purposes have been modified to better reflect the needs of an Ontario public health unit. The new syndrome categories are as follows: gastroenteritis, respiratory infectious, fever/influenza-like illness, asthma, dermatological infectious, neurologic infectious and severe infectious. ${ }^{14}$ Alerts are generated by the RODS recursive least squares algorithm and a modified Centres for Disease Control and Prevention Early Aberration Reporting System C1-like cumulative sum algorithm. ${ }^{17}$ The recursive least squares algorithm examines a longer time window and is designed to detect a slower, seasonal increase (e.g., influenza), while the cumulative sum algorithm is better suited for detection of a sudden sharp increase in counts (e.g., a GI outbreak). Two years of retrospective data were available for testing the algorithms and comparing alert periods. For real-time data, the algorithms are run every 6 hours, immediately upon completion of the count caching process. When an anomaly is detected, an email alert is automatically sent to designated users. This consists of a log entry containing the time, syndrome, count and the threshold that was exceeded to generate the alert. These log entries can be queried and viewed in the system. Anomaly detection is based on an observed to expected ratio 
and a predetermined threshold for a given geographical area, based on postal code.

\section{Spatial mapping}

Maps were created using the ESRI ArcIMS Geographic Information System services via the Internet. ${ }^{18}$ Because Canadian postal codes differ in urban and rural areas, RODS 3E was customized to handle urban and rural geography differently.

The last digit of the 6-digit postal code was dropped to maintain anonymity, and health units were used as the geographic unit for restricting counts (as opposed to counties in the United States). Because Ontario public health units have health promotion and disease surveillance mandates, several layers were added to the system, including geographic names, locations and contact information for towns and cities, schools, hospitals, major roads, long-term care homes, daycares and other facilities within jurisdictions. Further information describing technical implementation is available from the authors.

\section{Results and discussion}

\section{Preliminary results for main objectives}

Objective 1: to determine if real-time monitoring of ED patient chief complaints can assist in the early detection of outbreaks of GI and respiratory illnesses

Over the 2-year pilot project phase there were opportunities to assess the alerting and response capacity of the EDSS system. For example, retrospective analysis of influenza seasons has demonstrated respiratory syndrome alerts well in advance of documented influenza season. This has been used to prospectively monitor influenza activity and provide early notification and messaging with regard to precautions for front line health care workers. It also allows the hospital to prepare for surges of respiratory illness. The system also provides real-time information to public health personnel about outpatient visits and attack rates, facilitating rapid risk group assessments based on demographic and geographic distribution of illness, which could have a significant impact on pandemic planning. Providing front-line health care workers with surveillance information allows for improved viral culture detection, strain detection, monitoring for unusual severity of illness through CTAS and requests for cultures to identify circulating strains. The system has improved collaboration between public health and the acute care sector, and enhanced detection and management of other outbreaks, such as norovirus and food-borne Salmonella.
Details of these outbreaks are being prepared for future publications.

\section{Objective 2: to determine whether the surveillance tool can enhance public health investigation of disease out- breaks}

KFL\&A Public Health has collaboratively developed procedures for communication with participating hospitals, public health, laboratories, long-term care homes and other stakeholders to ensure timely appropriate response to system alerts. These protocols include steps to incorporate RODS $3 \mathrm{E}$ as a tool to aid our ongoing public health investigation of community or institutional outbreaks. Stakeholder communications consist of biweekly reports detailing GI and respiratory syndrome activity, ED volumes, admissions of interest and other relevant information such as institutional outbreaks, health alerts, seasonal information and applicable website links.

At the time of the pilot, KFL\&A Public Health was the only health unit in Ontario with an operational ED syndromic surveillance system. Contrary to many of our US colleagues who use multiple data sources for monitoring infectious disease activity, Ontario public health officials are limited to laboratory confirmed reportable disease data, which is often received long after symptom onset. Outbreaks have been an additional data source to support or deny the existence of alerts in the KFL\&A area. For example, during a Salmonella enteritidis outbreak in November and December 2005, KFL\&A was one of the first health units in Ontario to identify the source of the outbreak through real-time monitoring and subsequent linking with front-line health care workers for appropriate identification and culturing of suspect cases. Stakeholders (i.e., the public health system, laboratories, hospital EDs, infection control and management) who receive regular communication via the biweekly reports were surveyed using an online survey to indicate the usefulness of the reports. In addition to welcoming the information detailing infectious disease activity, they appreciated the exchange of information between public health and acute care.

\section{Objective 3: to address whether or not the tool can improve the capacity of public health professionals to respond to alerts of infectious disease outbreaks}

The inclusion of innovative data elements (e.g., CTAS and FRI) within the system allows for better characterization of outbreak severity and enables more effective resource allocation within acute care settings. ${ }^{19}$ Jiminez and colleagues found that the CTAS scale was a valid instrument for predicting admission rates, hospital length of stay and diagnostic 
use. ${ }^{10}$ Further studies have revealed that it correlates well with length of stay and is a significant factor in predicting physician workload. ${ }^{12}$ Hence, we are assessing its use as a marker of disease severity for patients in the same syndrome class. FRI screening has continued at most facilities in Ontario and this screening tool is incorporated into the Ontario Health Plan for an Influenza Pandemic (OHPIP). ${ }^{20}$ The inclusion of the FRI screening tool ensures compliance with the pandemic plan surveillance requirements in a seamless fashion, negating any change in practice or increased workload for front line health care workers.

By expanding acute care surveillance, KFL\&A Public Health has achieved a broader, more timely picture of community infectious disease activity than was previously possible, particularly for the more severe infections requiring ED assessment. This additional surveillance piece is essential for the potential early warning of new and emerging infections, as well as being a key component of local pandemic planning. Electronic collection of disease activity levels, subpopulations at risk, FRI and hospitalizations allows automated monitoring of the extent and severity of the influenza season, which can inform public health response and management strategies. This capacity has not previously been available in public health units.

\section{Secondary uses}

The system allows real-time tracking of admissions for sentinel diseases (e.g., meningitis and encephalitis), influenzalike illness or unusual increases in a particular demographic group or symptom presentation. It also offers the capacity to monitor injuries, mental health presentations and other chronic diseases such as asthma. Future potential uses include linking patient presentations with environmental heat or smog alerts, sentinel event surveillance and disaster medicine. These areas require further evaluation.

\section{Evaluation}

A comprehensive 3-part evaluation was performed (process-outcome, cost-benefit and technical) to assess the utility of an early warning system within a public health unit designed for real-time surveillance of infectious disease events. ${ }^{21}$ These reports were presented to the Syndromic Surveillance Steering Committee of the MOHLTC Public Health Division.

\section{Conclusion}

Real-time linkages between emergency departments and public health can provide information that enhances outbreak detection, response and recovery. The surveillance system described here proved useful in seasonal influenza and food-borne outbreaks, and similar systems show promise in terms of monitoring diverse emergent and disaster situations, although further work is required to refine syndrome definitions, improve data quality, and optimize sensitivity and specificity for anomaly detection and alerting. We are now considering expansion to other areas of the province, assessing the utility of the RODS 3E tool for disaster and emergency planning, and exploring the possibility of using this tool to monitor additional information sources, including Telehealth (Ontario's telephone health hotline), Occupational Health and Safety data from participating hospitals and over-the-counter pharmaceutical sales alerts from the Canadian Network for Public Health Intelligence's Alternative Surveillance Alert Project.

Acknowledgements: This Project was funded by the Public Health Division of the Ontario Ministry of Health and Long-Term Care with significant support from Kingston, Frontenac, Lennox and Addington Public Health, Kingston General Hospital, Hotel Dieu Hospital, Quinte Health Care Hospitals and Queen's University. In particular, we wish to acknowledge the efforts of the Project Steering Committee and Marlon Drayton and Kristie Willson from the Public Health Division who have supported us in the development and advancement of this project.

Competing interests: None declared.

\section{References}

1. Walker D. For the public's health: initial report of the Ontario Expert Panel on SARS and Infectious Disease Control. Toronto $(\mathrm{ON})$ : Ministry of Health and Long-Term Care; 2003.

2. The National Advisory Committee on SARS and Public Health. Learning from SARS: renewal of public health in Canada. Ottawa (ON): Health Canada; 2003.

3. Campbell A. The SARS Commission - spring of fear - final report. Toronto (ON): Ontario Ministry of Health and LongTerm Care; 2006. Available: www.health.gov.on.ca/english /public/pub/ministry_reports/campbell06/campbell06.html (accessed 2007 Feb 20).

4. Ministry of Health and Long-Term Care. Operation health protection: an action plan to prevent threats to our health and to promote a healthy Ontario. 2004. Available: www.health.gov.on.ca/english /providers/project/ohp/ohp_mn.html (accessed 2007 Feb 20).

5. Buehler JW, Hopkins RS, Overhage JM, et al.; CDC Working Group. Framework for evaluating public health surveillance systems for early detection of outbreaks: recommendations from the CDC Working Group. MMWR Recomm Rep 2004;53 (RR-5):1-11.

6. Espino JU, Wagner C, Szczepaniak F-C, et al. Removing a barrier to computer-based outbreak and disease surveillance - the RODS Open Source Project. MMWR Morb Mortal Wkly Rep 2004;53:32-9. 
7. Ontario Ministry of Health and Long-Term Care. Emergency department chief complaint syndromic surveillance system pilot project: technical evaluation. Kingston $(\mathrm{ON})$ : The Ministry; 2006.

8. Canadian Association of Emergency Physicians. Implementation guidelines for the Canadian Emergency Department Triage and Acuity Scale (CTAS). Version: CTAS16 December 16, 1998. Available: www.caep.ca/CMS/get_file.asp?id=d09ba8ebe 7b649338e35c69dacc5c1f8\&ext=.pdf\&name=ctased16.pdf $($ accessed 2007 Feb 20).

9. Murray M, Bullard M, Grafstein E; CTAS and CEDrosoph Inf Serv National Working Groups. Revisions to the Canadian Emergency Department Triage and Acuity Scale Implementation Guidelines. CJEM 2004;6:421-7.

10. Jimenez JG, Murray MJ, Beveridge R, et al. Implementation of the Canadian Emergency Department Triage and Acuity Scale in the Principality of Andorra: Can triage parameters serve as emergency department quality indicators? CJEM 2003;5:315-22.

11. Yoon P, Steiner I, Reinhardt G. Analysis of factors influencing length of stay in the emergency department. CJEM 2003;5:155-62.

12. Innes GD, Stenstrom R, Grafstein E, et al. Prospective time study derivation of emergency physician workload predictors. CJEM 2005;7:299-308.

13. Ontario Ministry of Health and Long-Term Care. Preventing respiratory illnesses protecting patients and staff: infection control and surveillance standards for febrile respiratory illness (FRI) in non-outbreak conditions in acute care hospitals. 2003. Available: www.health.gov.on.ca/english/providers/program /pubhealth/sars/docs/docs3/dir_infec_control_010604.pdf (accessed 2007 Feb 16).

14. O'Connor K, Rimmer M, Moore K, et al. Maximum entropy models in chief complaint classification. Adv Dis Surv 2007;2:23.
15. Chapman WW, Dowling JN, Wagner MM. Classification of emergency department chief complaints into 7 syndromes: a retrospective analysis of 527,228 patients. Ann Emerg Med 2005; 46:445-55.

16. Chapman WW, Dowling JN, Ivanov O, et al. Evaluating natural language processing applications applied to outbreak and disease surveillance. Paper presented at: Proc 36th Symposium on the Interface: Computing Science and Statistics; 26 May, 2004; Baltimore, Maryland.

17. Hutwagner L, Browne T, Seeman GM, et al. Comparing aberration detection methods with simulated data. Emerg Infect Dis 2005;11:314-6.

18. ESRI ArcIMS. California: Environmental Systems Research Institute, Inc. (ESRI). Available: www.esri.com/software/arcgis /arcims/index.html (accessed 2007 Apr 10).

19. Moore K, Rimmer M, O'Connor K, et al. Integration of hospital admissions, febrile respiratory illness screening and triage acuity score into an emergency department syndromic surveillance system. Adv Dis Surv 2006;1:51.

20. Ontario Ministry of Health and Long-Term Care Provincial Infectious Disease Advisory Committee. Preventing respiratory illnesses; protecting patients and staff. Best practices in surveillance and infection prevention and control for febrile respiratory illness (FRI), excluding tuberculosis, for all Ontario health care settings (revised edition). Toronto (ON): The Ministry; 2006.

21. Moore K, Rimmer M, O'Connor K, et al. Multi-sectored approach to evaluation of a syndromic surveillance system. Adv Dis Surv 2006;1:52.

Correspondence to: Dr. Kieran Moore, Queen's University Department of Emergency Medicine, c/o Kingston General Hospital, 76 Stuart St, Kingston ON K7L 2V7; moorek1@kgh.kari.net 